\title{
Cirugía de Pouch Ileal en tres tiempos: ¿por qué vale la pena?
}

\author{
David M. Schwartzberg, Patricio B. Lynn \\ Assistant Professor of Surgery, Zucker School of Medicine, Hofstra-Northwell Health, NY. \\ General Surgery Division, NYU Langone Health, New York, NY.
}

La Coloproctectomía total con pouch ileal es el procedimiento de elección en pacientes con colitis ulcerosa (CU), poliposis adenomatosa familiar (PAF) y en casos seleccionados de enfermedad de Crohn (EC). En los Estados Unidos (EE. UU.) las tasas de enfermedad inflamatoria intestinal (EII) están aumentando y, a pesar de los avances médicos, los nuevos agentes biológicos y la aparición de grupos multidisciplinarios de EII, aproximadamente un tercio de los pacientes con CU requieren cirugía en algún momento de la vida. ${ }^{1}$

Actualmente, en centros especializados la cirugía de pouch ileal es exitosa en más del $95 \%$ de los casos. ${ }^{2} \mathrm{El}$ éxito del pouch ileal se mide en calidad de vida y depende de varios factores, siendo las complicaciones perioperatorias los principales determinantes del funcionamiento a largo plazo, especialmente las complicaciones sépticas secundarias a una dehiscencia anastomótica. ${ }^{3}$

Por este motivo, la cirugía del pouch ileal ha sido desarrollada en etapas minimizando la morbilidad y optimizando la condición del paciente. Esto es especialmente importante en pacientes operados de urgencia, la mayoría desnutridos y bajo tratamientos inmunosupresores.

Los tres tiempos consisten en colectomía total con ileostomía terminal, seguido por proctectomía e ileostomía en asa 6 meses luego de la colectomía. Finalmente, el cierre de ileostomía se realiza a los tres meses de la proctectomía, previo estudio radiológico con contraste hidrosoluble mostrando indemnidad del pouch. Alternativas menos conservadoras incluyen la cirugía en dos tiempos (proctocolectomía con pouch ileal e ileostomía en asa, seguido de cierre de ileostomía), cirugía en dos tiempos modificada (colectomía total con ileostomía terminal, seguido de proctocolectomía con pouch ileal) y finalmente la cirugía en un tiempo (proctocolectomía total con pouch ileal). En la literatura, la frecuencia con la que el abordaje en tres tiempos es utilizado varía mucho con reportes que van desde el 11 hasta el $69 \%$ de los casos; ${ }^{4,5}$ lo cual sugiere que la elección del abordaje está determinada más por el centro en cuestión y su escuela quirúrgica que por las características del paciente.

\footnotetext{
Los autores declaran ausencia de conflictos de interés .

Patricio B. Lynn

patricio.lynn@nyulangone.org

Recibido: mayo de 2020. Aceptado: junio de 2020.
}

En cuanto a los resultados funcionales, las series coinciden en que la cirugía en tres tiempos tiene iguales o mejores resultados que la cirugía en dos tiempos. ${ }^{4,6-8}$ Algo similar ocurre con las complicaciones postoperatorias y las tasas de sepsis pélvica. La mayoría de las series, con escasas excepciones, reportan resultados favorables a la cirugía en tres tiempos o similares entre los dos abordajes. La tabla 1 resume los resultados de las series más representativas.

Un estudio retrospectivo de 2013 con 147 pacientes a lo largo de 10 años de experiencia demostró que la cirugía en tres tiempos tuvo menos complicaciones postoperatorias que la cirugía en dos tiempos, sin embargo, ambos abordajes tuvieron las mismas tasas de falla/pérdida del pouch. Curiosamente, una actualización de esta serie, ahora con 212 pacientes, no encontró una mayor asociación con complicaciones por parte de la cirugía en dos tiempos. Analizando detalladamente los datos, si bien la diferencia no fue estadísticamente significativa los pacientes operados en dos tiempos tuvieron casi el doble de dehiscencias anastomóticas que los operados en tres $(9.6$ vs. $5 \%) .{ }^{9}$ A pesar de esto, los resultados funcionales fueron similares entre los grupos.

Ante la ausencia de estudios prospectivos controlados y randomizados, la mejor evidencia disponible probablemente sea la obtenida a través de análisis utilizando la base de datos del National Surgical Quality Improvement Program del American College of Surgeons. Dos estudios con más de 2000 pacientes son los más significativos. El primero, de 2015, revisó las tendencias de la cirugía de pouch ileal en EE.UU., desde 2005 hasta 2011. Los autores demostraron que más del $70 \%$ de los procedimientos incluidos en la base de datos se realizaron en dos tiempos. La tasa de complicaciones fue similar 11.5 vs. 9.4\% p=0.1. No obstante, el perfil de las complicaciones fue diferente. Mientras que los pacientes operados en tres tiempos tuvieron más infecciones de la herida quirúrgica (11.5 vs. $7.3 \% \mathrm{p}<0.01)$, los pacientes operados en dos tiempos tuvieron más infecciones intraabdominales/pélvicas ( 9.4 vs. $6.7 \% \mathrm{p}=0.05$ ) y requirieron más reoperaciones (8 vs. $4.4 \%$ $\mathrm{p}<0.01)$. El mismo estudio también demostró que los pacientes operados en tres tiempos recibieron menos corticoides perioperatorios, tuvieron una menor pérdida de peso, mejores niveles de albúmina y menos sepsis preope- 
TABLA 1: RESULTADOS DE SERIES COMPARANDO CIRUGÍA DE POUCH ILEAL EN DOS VS. TRES TIEMPOS

\begin{tabular}{|c|c|c|c|c|c|}
\hline Autor & Año & $n$ & $3 \mathrm{~T}(\%)$ & $\begin{array}{l}\text { Complicaciones/ } \\
\text { Sepsis }\end{array}$ & $\begin{array}{l}\text { Función } \\
\text { evacuatoria }\end{array}$ \\
\hline Nicholls $^{6}$ & 1989 & 152 & $62 \%$ & Sin diferencia & Mejor 3T \\
\hline Galandiuk $^{4}$ & 1991 & 871 & $11 \%$ & $\begin{array}{l}\text { 3T más complicaciones sépticas, } \\
\text { menos obstrucción. }\end{array}$ & $\begin{array}{l}\text { Resultados } \\
\text { similares }\end{array}$ \\
\hline Penna $^{7}$ & 1993 & 156 & $50 \%$ & 2T más complicaciones y reoperaciones & Mejor 3Т \\
\hline Heustchen $^{13}$ & 2002 & 554 & $29 \%$ & Sin diferencia a 1 y 3 años de seguimiento & NR \\
\hline Swenson ${ }^{8}$ & 2005 & 54 & $57 \%$ & Sin diferencia & $\begin{array}{l}\text { Resultados } \\
\text { similares }\end{array}$ \\
\hline $\operatorname{Lim}^{15}$ & 2007 & 335 & NR & Sin diferencia & NR \\
\hline Hicks $^{16}$ & 2013 & 144 & $19.4 \%$ & $2 \mathrm{~T}$ más complicaciones, $=$ tasa de DA & NR \\
\hline $\mathrm{Gu}^{5}$ & 2013 & 588 & $69 \%$ & Sepsis 2 T $18 \%$ vs $3 T$ T $\%$ & NR \\
\hline Bikhchandani ${ }^{10}$ & 2015 & 2002 & $27.5 \%$ & Sin diferencia & NR \\
\hline Kochar11 & 2018 & 2395 & $34 \%$ & $\begin{array}{l}\text { 3T menos reoperaciones y menos } \\
\text { complicaciones }\end{array}$ & NR \\
\hline Lee $^{9}$ & 2019 & 212 & $25.9 \%$ & Sin diferencia & $\begin{array}{l}\text { Resultados } \\
\text { similares }\end{array}$ \\
\hline
\end{tabular}

*Referencias: 3T: cirugía en tres tiempos, 2T: cirugía en dos tiempos, NR: no reportado, DA: dehiscencia anastomótica.

ratorias al momento de la cirugía de pouch. ${ }^{10}$ En otras palabras, al momento de la cirugía del pouch los pacientes operados en tres tiempos estaban en mejores condiciones generales. El segundo estudio en cuestión analizó la misma base de datos desde 2011 hasta 2015. Los autores compararon la incidencia de reoperaciones y complicaciones en pacientes sometidos a cirugía de pouch al momento de la colectomía vs. pacientes en los cuales la confección del pouch fue demorada. Con 2300 pacientes, 34\% fue tratado con cirugía demorada; estos últimos presentaron significativamente menos reoperaciones así como complicaciones durante los primeros 30 días postoperatorios. $^{11}$

La realidad es que, al realizar más intervenciones, mayor será el tiempo que los pacientes estarán con una ileostomía y cada intervención tiene sus propias complicaciones (intubación endotraqueal, infecciones del sitio quirúrgico, dolor postoperatorio, internaciones y costos) las cuales deben ser discutidas en cada caso. A pesar de esto, varias son las ventajas de un abordaje en tres tiempos:

1. el colon puede ser analizado obteniendo un diagnóstico preciso de CU o EC así como displasia y eventualmente cáncer (que podría requerir tratamiento adyuvante) previo a la proctectomía,

2. el mesenterio sufre cierto grado de estiramiento con la ileostomía terminal (lo que permitiría una mejor confección del pouch),

3. la ileostomía en asa minimiza las complicaciones de una posible dehiscencia anastomótica y
4. la proctectomía junto con su morbilidad es demorada por tres meses permitiendo una mejor preparación física y mental del paciente antes de la confección del pouch.

Los pacientes que reciben biológicos constituyen un particular grupo de riesgo. Si bien reportes tempranos no encontraron relación entre el uso de biológicos y complicaciones postoperatorias, ${ }^{12,13}$ un estudio más reciente de la Cleveland Clinic, de 2013, con 588 pacientes demostró que el uso de agentes biológicos es una variable independiente asociada al desarrollo de sepsis pélvica en el análisis multivariado (HR 2.62; $\mathrm{p}=0.02) .{ }^{5}$ Coincidiendo con esto, el grupo de la Universidad de Cornell utilizando una base de datos del Departamento de salud del Estado de Nueva York realizó un estudio retrospectivo con poco más de 7000 pacientes. Los autores demostraron que, desde que el Infliximab fue aprobado, los pacientes con CU no solo continuaron requiriendo cirugía, sino que en la era post-infliximab sufrieron más y mayores complicaciones. ${ }^{14}$ Esta debilitada población es precisamente el grupo que, a nuestro criterio, más se beneficia de un procedimiento en tres tiempos.

Estas consideraciones llevaron a que indiquemos la cirugía en tres tiempos en prácticamente todos los casos. La posibilidad de una complicación séptica con sus consecuencias funcionales es un precio muy alto a pagar por el paciente y es responsabilidad del cirujano optimizar las condiciones para minimizar la morbilidad de un procedimiento ya de por sí complejo (Tabla 1). 


\section{BIBLIOGRAFÍA}

1. Facts about inflammatory bowel diseases. Crohns and Colitis Foundation. Available at: https://site.crohnscolitisfoundation.org/ resources/facts- about-inflammatory.html

2. Gorgun E, Remzi FH. Complications of ileoanal pouches. Clinics 2004 Feb;17:43-55.

3. Kiely JM, Fazio VW, Remzi FH, Shen B, Kiran RP. Pelvic sepsis after IPAA adversely affects function of the pouch and quality of life. Dis Colon Rectum 2012;55:387-92.

4. Galandiuk S, Pemberton JH, Tsao J, Ilstrup DM, Wolff BG. Delayed ileal pouch-anal anastomosis. Complications and functional results. Dis Colon Rectum 1991;34:755-58.

5. Gu J, Remzi FH, Shen B, Vogel JD, Kiran RP. Operative strategy modifies risk of pouch-related outcomes in patients with ulcerative colitis on preoperative anti-tumor necrosis factor- $₫$ therapy. Dis Colon Rectum 2013;56:1243-52.

6. Nicholls RJ, Holt SD, Lubowski DZ. Restorative proctocolectomy with ileal reservoir. Comparison of two-stage vs. three-stage procedures and analysis of factors that might affect outcome. Dis Colon Rectum 1989;32:323-26.

7. Penna C, Daude F, Parc R, Tiret E, Frileux P, Hannoun L, et al. Previous subtotal colectomy with ileostomy and sigmoidostomy improves the morbidity and early functional results after ileal pouch-anal anastomosis in ulcerative colitis. Dis Colon Rectum 1993;36:343-48.

8. Swenson BR, Hollenbeak CS, Poritz LS, Koltun WA. Modified two-stage ileal pouch-anal anastomosis: Equivalent outcomes with less resource utilization. Dis Colon Rectum 2005;48:256-61.

9. Lee GC, Deery SE, Kunitake H, Hicks CW, Olariu AG, Savitt LR, et al. Comparable perioperative outcomes, long-term outcomes, and quality of life in a retrospective analysis of ulcerative colitis patients following 2-stage versus 3-stage proctocolectomy with ileal pouchanal anastomosis. Int J Colorectal Dis 2019;34:491-99.

10. Bikhchandani J, Polites SF, Wagie AE, Habermann EB, Cima RR. National trends of 3- versus 2-stage restorative proctocolectomy for chronic ulcerative colitis. Dis Colon Rectum 2015;58:199-204.

11. Kochar B, Barnes EL, Peery AF, Cools KS, Galanko J, Koruda $\mathrm{M}$, et al. Delayed ileal pouch anal anastomosis has a lower 30-day adverse event rate: Analysis from the National Surgical Quality Improvement Program. Inflamm Bowel Dis 2018;24:1833-39.

12. Gainsbury ML, Chu DI, Howard LA, Coukos JA, Farraye FA, Stucchi AF, et al. Preoperative infliximab is not associated with an increased risk of short-term postoperative complications after restorative proctocolectomy and ileal pouch-anal anastomosis. J Gastrointest Surg 2011;15:397-403.

13. Yang Z, Wu Q, Wang F, Wu K, Fan D. Meta-analysis: effect of preoperative infliximab use on early postoperative complications in patients with ulcerative colitis undergoing abdominal surgery. Aliment Pharmacol Ther 2012;36:922-28.

14. Abelson JS, Michelassi F, Mao J, Sedrakyan A, Yeo H. Higher surgical morbidity for ulcerative colitis patients in the era of biologics. Ann Surg 2018;268:311-17.

15. Lim M, Sagar P, Abdulgader A, Thekkinkattil D, Burke D. The impact of preoperative immunomodulation on pouch-related septic complications after ileal pouch-anal anastomosis. Dis Colon Rectum 2007;50:943-51.

16. Hicks CW, Hodin RA, Bordeianou L. Possible overuse of 3-stage procedures for active ulcerative colitis. JAMA Surg 2013;148:65864. 